\title{
Pimozide Inhibits the AcrAB-TolC Efflux Pump in Escherichia coli
}

\author{
Jürgen A. Bohnert*, Sabine Schuster and Winfried V. Kern
}

Center for Infectious Diseases and Travel Medicine, University Hospital, and Department of Medicine, Albert-LudwigsUniversity, Freiburg, Germany

\begin{abstract}
Efflux pump inhibitors (EPIs) are attractive compounds to reverse multidrug-resistance in clinically relevant bacterial pathogens. In this study we tested the ability of the neuroleptic drug pimozide to inhibit the Escherichia coli AcrAB-TolC efflux pump, whose overproduction confers resistance to various antimicrobial agents. A real-time Nile red efflux assay in the AcrAB - overproducing strain 3-AG100 revealed that pimozide was capable of full inhibition of this pump at a concentration of $100 \mu \mathrm{M}$, which is far below its intrinsic MIC $(>1 \mathrm{mM})$. However, MIC assay demonstrated very little effect of pimozide with regard to reduction in MICs of various antimicrobial compounds.

Only oxacillin MICs were reduced twofold in the presence of pimozide at 100 and $200 \mu \mathrm{M}$.

Since pimozide did considerably enhance accumulation of ethidium bromide in a fluorescence assay, ethidium bromide MIC assays in the presence and absence of this putative EPI were performed. They revealed that pimozide was able to reduce the MICs of ethidium bromide by 4-fold. In line with previous reports we suggest that the capability of EPIs to restore the susceptibility to antimicrobial agents can be highly substrate-specific due to different substrate binding sites.
\end{abstract}

Keywords: AcrB, efflux pump inhibitor, multidrug resistance, pimozide.

\section{INTRODUCTION}

The Escherichia coli AcrB multidrug efflux pump is a membrane protein that recognizes many chemically unrelated compounds including various dyes and antibiotics and expels them in collaboration with the so called membrane fusion protein AcrA and the outer membrane protein TolC which are both needed for function. The pump belongs to the resistance-nodulation-division (RND) family of efflux pumps that are common in gram-negative microorganisms. Based on various $\mathrm{X}$-ray crystallographic, mutagenic and biochemical studies the tripartite efflux pump AcrAB-TolC is one of the best characterized bacterial efflux pumps [1-10]. It is also the only RND pump in E. coli that contributes significantly to antibiotic resistance against clinically relevant antibiotics due to its constitutive expression. Exposure to antimicrobial agents can further increase efflux-mediated resistance due to mutations in the acrR or marR gene leading to upregulation of the efflux pump [11]. Since the above mutations can relatively rapidly be selected by exposure to only one antibiotic and since the substrate spectrum of AcrABTolC is so large, generation of multidrug resistance via efflux of antimicrobial compounds is a real threat to treatment options in the clinic.

Since the antimicrobial drug pipeline is relatively empty - especially against gram-negatives - efflux pump inhibitors (EPIs) that revert multidrug resistance are an attractive target. Interestingly, several working groups have recently described psychotropic drugs, namely phenothiazines $[12,13]$

*Address correspondence to this athour at the University Hospital, Hugstetter Strasse 55, D-79106 Freiburg, Germany; Tel: +49-761- 270 18190; Fax: ++49-761-270 18200; E-mail: juergen@bohnert.name and selective serotonine reuptake inhibitors (SSRIs) [14] that are capable of inhibiting the AcrAB-TolC efflux pump in E. coli. By screening more psychotropic drugs we discovered that the neuroleptic drug pimozide is also a model efflux pump inhibitor.

\section{MATERIALS AND METHODS}

\section{Bacterial Strains and Growth Conditions}

E. coli strains 3-AG100 (a multidrug-resistant mutant (gyrA marR) with acrB overexpression obtained from E. coli K-12 strain AG100 after repeated exposure to a fluoroquinolone) and the $a c r B$ knockout strain 1-DC14 [15] were grown in LB broth ( $1 \%$ tryptone, $0.5 \%$ yeast extract, and $1 \%$ $\mathrm{NaCl}$ ), or on LB agar (1.5\%) plates (Roth, Karlsuhe, Germany).

\section{Chemicals}

All chemicals were obtained from Sigma-Aldrich (Taufkirchen, Germany). Since pimozide is poorly soluble in water it was dissolved in hot lactic acid $(0.03 \%)$.

\section{Susceptibility Testing}

The MICs of a range of antimicrobial agents in the presence and absence of pimozide were determined in a 96-well microtiter plate using strains incubated overnight at $37^{\circ} \mathrm{C}$ in a volume of $100 \mu 1 /$ well by a standard LB broth microdilution procedure and a final inoculum of $5 \times 10^{5} \mathrm{CFU} / \mathrm{ml}$. MIC testing was done in triplicate. Custom 96-well microtiter plates containing selected antimicrobials at increasing concentrations were purchased from Merlin Diagnostics (Bornheim, Germany). 
Table 1. Synergy of Pimozide with Selected Antibacterial Drugs and Ethidium in E. coli.

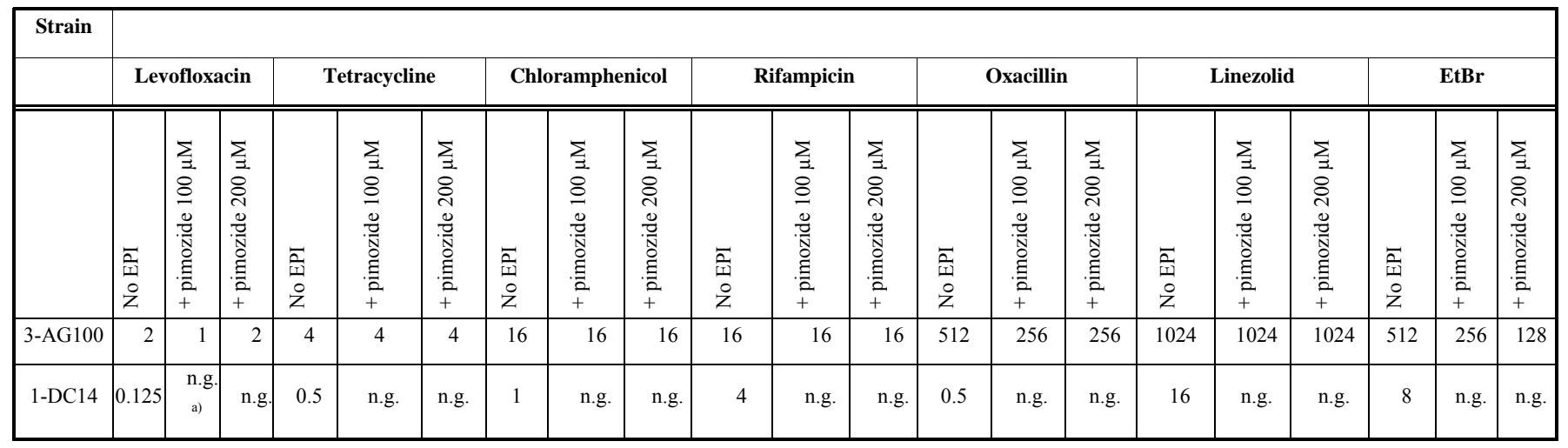

a) no growth.

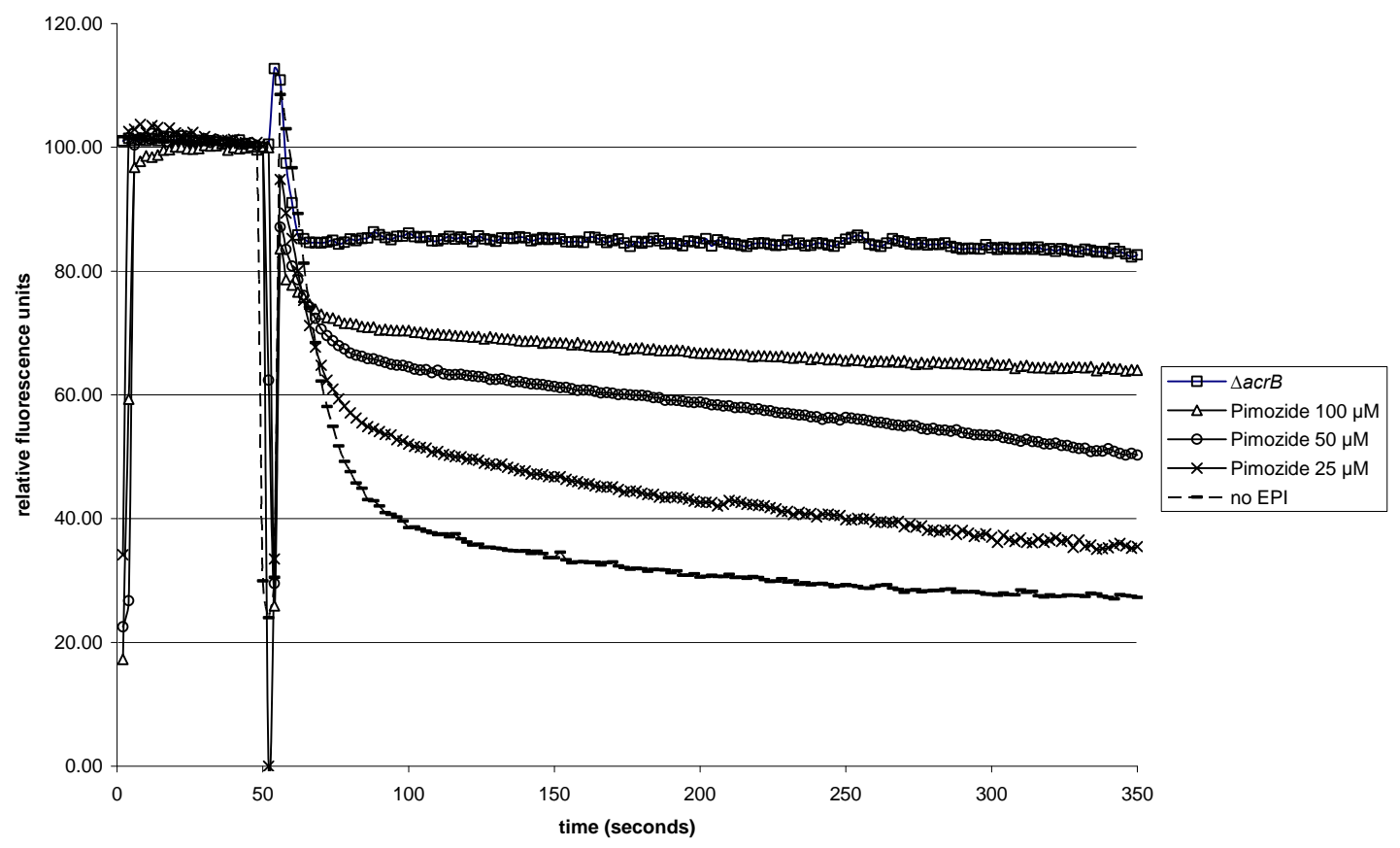

Fig. (1). Dose-dependent Nile red efflux inhibition in 3-AG100 in the presence of pimozide ( $\triangle a c r B$ strain 1-DC14 used for comparison). Addition of glucose (final concentration $50 \mathrm{mM}$ ) 50 seconds into the experiment to trigger efflux.

\section{RESULTS}

\section{Fluorescence Assays}

The protocols for the Nile red efflux [16] and ethidium bromide (EtBr) accumulation [17] assay have been published previously. The only modification was that the standard 20 $\mathrm{mM}$ potassium phosphate buffer $(\mathrm{pH} 7)$ amended with $1 \mathrm{mM}$ $\mathrm{MgCl}_{2}$ used in the Nile red efflux assay was also used in the ethidium bromide accumulation assay. Moreover, the $\mathrm{EtBr}$ concentration was increased to $10 \mu \mathrm{M}$.

Using AcrAB-TolC overproducing strain 3-AG100 pimozide at concentrations of up to $200 \mu \mathrm{M}$ did not reduce the MIC of the following antibiotics: tetracycline, chloramphenicol, rifampicin, and linezolid (Table 1). Oxacillin MICs were reduced twofold at $200 \mu \mathrm{M}$ but not at $100 \mu \mathrm{M}$. The levofloxacin MICs behaved somewhat paradoxical with twofold reduction at $100 \mu \mathrm{M}$ but no reduction at $200 \mu \mathrm{M}$.
Adding the dye EtBr to the MIC panel, we determined that $\mathrm{EtBr}$ MICs were reduced 4-fold. The intrinsic MIC for pimozide was found to be $>1000 \mu \mathrm{M}$, whereas in the AcrBdeficient strain 1-DC14 it was $100 \mu \mathrm{M}$.

\section{Nile Red Efflux and EtBr Accumulation Assays}

Notable retardation of Nile red efflux in strain 3-AG100 was observed in the presence of pimozide at a threshold concentration of $25 \mu \mathrm{M}$ (Fig. 1). Adding higher concentration of pimozid led to increasing efflux retardation in a dosedependent manner. At $100 \mu \mathrm{M}$ Nile red efflux was almost completely abolished.

Adding the pimozide just $50 \mathrm{~s}$ before energization yielded the same results. Controls with $0.03 \%$ lactic acid and no pimozide did not alter the substrate efflux.

In line with these observations, $100 \mu \mathrm{M}$ pimozide led also to a pronounced increase in $\mathrm{EtBr}$ accumulation that was 


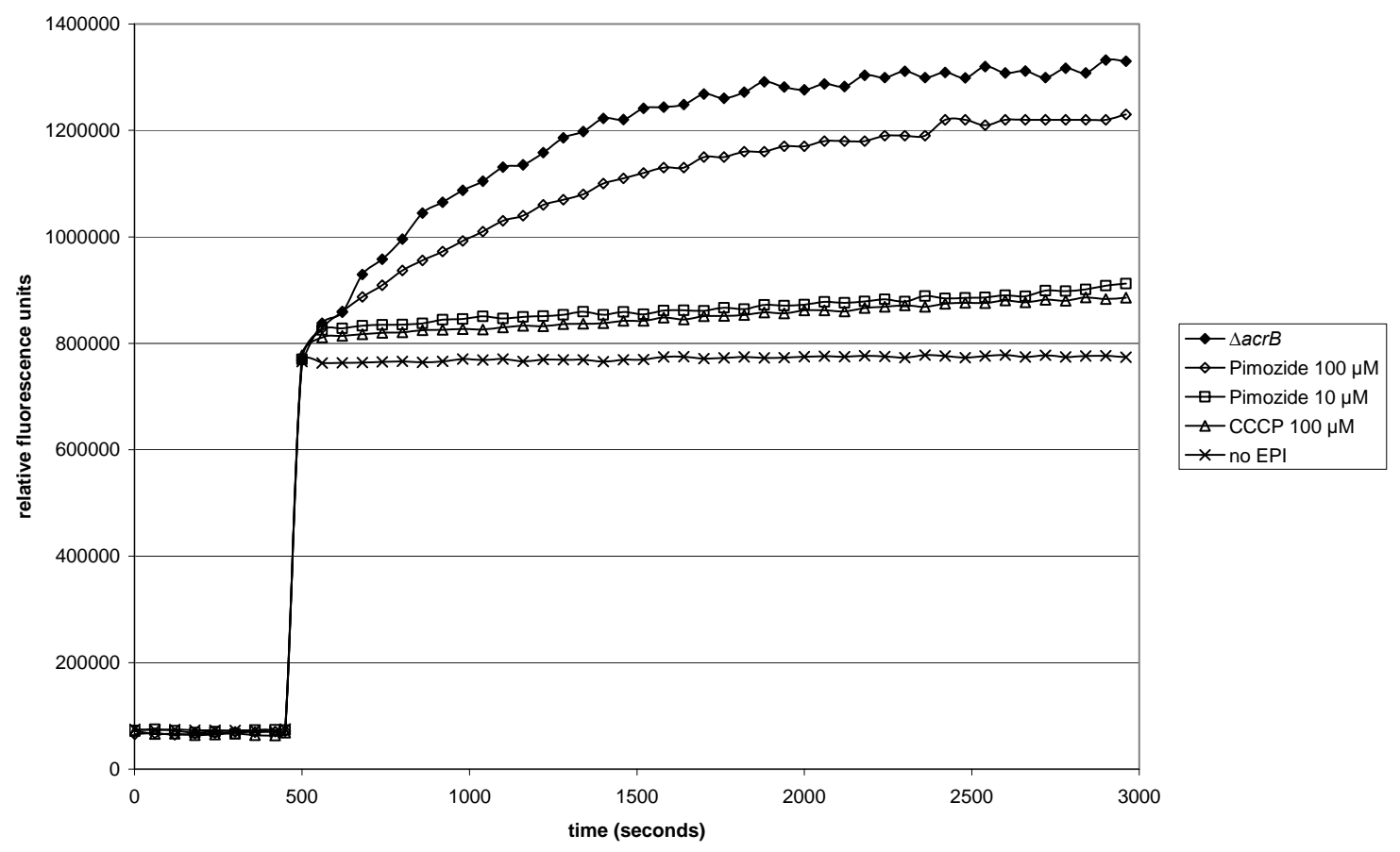

Fig. (2). Effect of pimozide on EtBr accumulation in the acrAB-overexpressing strain 3-AG100 ( $\triangle a c r B$ strain 1-DC14 used for comparison). Pimozide or CCCP were added 50 seconds and $\mathrm{EtBr}$ (final concentration $10 \mu \mathrm{M}$ ) 500 seconds into the experiment. $50 \mathrm{mM}$ glucose was present in the buffer throughout the assay.

even stronger than $100 \mu \mathrm{M}$ carbonyl cyanide $m$-chlorophenyl hydrazone (CCCP) (Fig. 2).

\section{DISCUSSION}

In the Nile red efflux and EtBr accumulation assays the neuroleptic drug pimozide behaved like an EPI, in the case of Nile red comparable to Phe-Arg- $\beta$-naphthylamine (PAßN) [18]. However, the effect in the MIC assays was generally much less pronounced. Only in the case of EtBr could a fourfold reduction in MIC be observed. It thus seems that the effect of pimozide is highly substrate-specific. Such behaviour has been described before in the well-studied EPI PAßN. Although this EPI potentiates the activity of a wide variety of antimicrobial compounds it fails to do so with ethidium bromide [19]. As recently suggested by molecular docking [20] and X-ray crystallographic [8] studies we hypothesize that the observed discrepancy is mainly due to different EPI and substrate binding sites so that the activity of a given EPI might be highly substrate specific. It seems that pimozide is an extreme case in this regard since we could only demonstrate that it interferes with the transport of $\mathrm{EtBr}$ and Nile red but not with a range of clinically relevant antimicrobials.

While this observation and the fact that the plasma peak levels of this drug required for EPI activity cannot be safely reached in humans preclude its clinical usefulness, we suggest that pimozide can still be valuable as a "narrowspectrum" model EPI to learn more about substrate - EPI interactions.

\section{CONFLICT OF INTEREST}

The authors confirm that this article content has no conflicts of interest.

\section{ACKNOWLEDGEMENTS}

Declared none.

\section{REFERENCES}

[1] Bohnert JA, Schuster S, Seeger MA, Fahnrich E, Pos KM, Kern WV. Site-directed mutagenesis reveals putative substrate binding residues in the Escherichia coli RND efflux pump AcrB. J Bacteriol 2008; 190: 8225-9.

[2] Bohnert JA, Schuster S, Szymaniak-Vits M, KernWV. Determination of real-time efflux phenotypes in Escherichia coli AcrB binding pocket phenylalanine mutants using a 1,2 '-Dinaphthylamine Efflux Assay. PloS ONE 2011; 6(6): e21196.

[3] Eicher T, Cha HJ, Seeger MA, et al. Transport of drugs by the multidrug transporter AcrB involves an access and a deep binding pocket that are separated by a switch-loop. Proc Natl Acad Sci USA 2012; 109: 5687-92.

[4] Husain F, Nikaido H. Substrate path in the AcrB multidrug efflux pump of Escherichia coli. Mol Microbiol; 2010; 78(2): 320-30.

[5] Koronakis V, Sharff A, Koronakis E, Luisi B, Hughes C. Crystal structure of the bacterial membrane protein TolC central to multidrug efflux and protein export. Nature 2000; 405: 914-9.

[6] Mikolosko J, Bobyk K, Zgurskaya HI, Ghosh P. Conformational flexibility in the multidrug efflux system protein AcrA. Structure 2006; 14: 577-87.

[7] Murakami S, Nakashima R, Yamashita E, Matsumoto T, Yamaguchi A. Crystal structures of a multidrug transporter reveal a functionally rotating mechanism. Nature 2006; 443: 173-9.

[8] Nakashima R, Sakurai K, Yamasaki S, Nishino K, Yamaguchi A. Structures of the multidrug exporter AcrB reveal a proximal multisite drug-binding pocket. Nature 2011; 480: 565-9.

[9] Seeger MA, Schiefner A, Eicher T, Verrey F, Diederichs K, Pos KM. Structural asymmetry of AcrB trimer suggests a peristaltic pump mechanism. Science 2006; 313: 1295-8.

[10] Takatsuka Y, Nikaido H. Covalently linked trimer of the AcrB multidrug efflux pump provides support for the functional rotating mechanism. J Bacteriol 2009; 191: 1729-37.

[11] Kern WV, Oethinger M, Jellen-Ritter AS, Levy SB. Non-target gene mutations in the development of fluoroquinolone resistance in Escherichia coli. Antimicrob Agents Chemother 2000; 44: 814-20. 
[12] Kristiansen JE, Thomsen VF, Martins A, Viveiros M, Amaral L. Non-antibiotics reverse resistance of bacteria to antibiotics. In Vivo 2010; 24: 751-4.

[13] Martins M, Dastidar SG, Fanning S, et al. Potential role of nonantibiotics (helper compounds) in the treatment of multidrugresistant Gram-negative infections: mechanisms for their direct and indirect activities. Int J Antimicrob Agents 2008; 31:198-208.

[14] Bohnert JA, Szymaniak-Vits M, Schuster S, Kern WV. Efflux inhibition by selective serotonin reuptake inhibitors in Escherichia coli. J Antimicrob Chemother 2011; 66: 2057-60.

[15] Jellen-Ritter AS, Kern WV. Enhanced expression of the multidrug efflux pumps AcrAB and AcrEF associated with insertion element transposition in Escherichia coli mutants Selected with a fluoroquinolone. Antimicrob Agents Chemother 2001; 45: 1467-72.

[16] Bohnert JA, Karamian B, Nikaido H. Optimized Nile Red efflux assay of AcrAB-TolC multidrug efflux system shows competition between substrates. Antimicrob Agents Chemother 2010; 54: 37705 .
[17] Bohnert JA, Kern WV. Selected arylpiperazines are capable of reversing multidrug resistance in Escherichia coli overexpressing RND efflux pumps. Antimicrob Agents Chemother 2005; 49: 84952.

[18] Renau TE, Leger R, Flamme EM, et al. Inhibitors of efflux pumps in Pseudomonas aeruginosa potentiate the activity of the fluoroquinolone antibacterial levofloxacin. J Med Chem 1999; 42: 492831 .

[19] Lomovskaya O, Warren MS, Lee A, et al. Identification and characterization of inhibitors of multidrug resistance efflux pumps in Pseudomonas aeruginosa: novel agents for combination therapy. Antimicrob Agents Chemother 2001; 45: 105-16.

[20] Takatsuka Y, Chen C, Nikaido H. Mechanism of recognition of compounds of diverse structures by the multidrug efflux pump AcrB of Escherichia coli. Proc Natl Acad Sci USA 2010; 107 : 6559-65.

(C) Bohnert et al.; Licensee Bentham Open.

This is an open access article licensed under the terms of the Creative Commons Attribution Non-Commercial License (http://creativecommons.org/licenses/by-nc/3.0/) which permits unrestricted, non-commercial use, distribution and reproduction in any medium, provided the work is properly cited. 